\section{Smith ScholarWorks}

$3-5-2010$

\section{Reactive Layer-by-Layer Assembly of Suspended Thin Films and Semipermeable Membranes at Interfaces Created Between Aqueous and Organic Phases}

\author{
Maren E. Buck \\ University of Wisconsin-Madison, mbuck@smith.edu \\ David M. Lynn \\ University of Wisconsin-Madison
}

Follow this and additional works at: https://scholarworks.smith.edu/chm_facpubs

Part of the Chemistry Commons

\section{Recommended Citation}

Buck, Maren E. and Lynn, David M., "Reactive Layer-by-Layer Assembly of Suspended Thin Films and Semipermeable Membranes at Interfaces Created Between Aqueous and Organic Phases" (2010). Chemistry: Faculty Publications, Smith College, Northampton, MA.

https://scholarworks.smith.edu/chm_facpubs/34 


\title{
Reactive Layer-by-Layer Assembly of Suspended Thin Films and Semipermeable Membranes at Interfaces Created Between Aqueous and Organic Phases
}

\author{
Maren E. Buck and David M. Lynn \\ M. E. Buck, Prof. D. M. Lynn Department of Chemistry University of Wisconsin-Madison 1101 \\ University Avenue, Madison, WI 53706 (USA)
}

\begin{abstract}
Suspended thin films of polymer (i.e., films or membranes that are not supported entirely by a solid substrate but rather are suspended across or within the open ends of channels, pores, or microcavities) are of interest in a broad range of fundamental and applied contexts. Thin films suspended across micropores or nanopores, for example, have been investigated as sensory membranes, ${ }^{[1-4]}$ as membranes within microfluidic devices, ${ }^{[5-7]}$ and as permeable or semipermeable membranes for both separations ${ }^{[8-11]}$ and the controlled administration of therapeutic agents. ${ }^{[12-14]}$ Here, we demonstrate a straightforward "reactive" layer-by-layer approach $^{[15]}$ to the fabrication of suspended, covalently crosslinked, and chemically reactive thin-film composites that makes use of liquid/liquid interfaces created between immiscible aqueous and organic phases as templates for film fabrication. Removal of these liquid templates post-fabrication yields porous and semipermeable membranes suspended across pores and orifices with sizes ranging from tens of micrometers to many millimeters in diameter. These suspended films are mechanically robust and can be used directly as membranes for the separation of mixtures of small and/or large molecules. Our results thus suggest the basis of a general and facile approach to the design and rapid fabrication of suspended polymer membranes of interest in the contexts of biotechnology, sensing, catalysis, and separations-based applications.
\end{abstract}

The layer-by-layer deposition of oppositely charged polymers on surfaces ${ }^{[16]}$ is a versatile method for the fabrication of nanostructured thin films (or "polyelectrolyte multilayers"). ${ }^{[17-20]}$ These methods permit precise control over film thickness and chemical composition, and they are useful for the assembly of thin films and composites on the surfaces of geometrically complex substrates. ${ }^{[17-20]}$ In general, solid substrates (planar surfaces, microparticles, etc.) serve as mechanical supports during layer-by-layer assembly and, often, as supports for the resulting films during use. ${ }^{[20]}$ However, there has been significant recent interest in the fabrication of both free-standing ${ }^{[2,3,9,21-26]}$ and/or suspended ${ }^{[2,3,9,21,22,24,26]}$ multilayer composites that could broaden significantly the range of applications for which these materials are suited. For example, the porosities and tuneable internal structures of polyelectrolyte multilayers present myriad opportunities for the design of suspended, flow-through membranes for catalysis, ${ }^{[11]}$ or the release and/or separation of chemical and biological species. ${ }^{[9,11,13,19]}$ Methods that permit the fabrication of multilayers directly across or within the openings of pores, channels, and microcavities would provide opportunities to exploit further the potentially useful properties of these materials.

\footnotetext{
(C) 2010 WILEY-VCH Verlag GmbH \& Co. KGaA, Weinheim

* Prof. D. M. Lynn Department of Chemical and Biological Engineering University of Wisconsin—Madison 1415 Engineering Drive, Madison, WI 53706 (USA)dlynn@engr.wisc.edu.
} 
Several groups have demonstrated that suspended films can be prepared by lifting multilayers first deposited on planar solid surfaces and then transferring these free-standing films onto arrays of microholes or cavities. ${ }^{[2,3,9,21,22]}$ This approach has been used successfully to separate mixtures of polymers and small molecules contained in microwell arrays ${ }^{[9]}$ and to characterize the physical and mechanical properties of suspended multilayers. ${ }^{[2,3,21,22]}$ However, the multistep processes required for this transfer-based approach prohibit (or at least significantly increase the difficulty of) fabricating suspended multilayers across or within the pores of topologically complex substrates (e.g., across the open ends of capillary tubes or micropipettes, or within the channels of microfluidic devices). In addition, the aqueous methods used to fabricate polyelectrolyte multilayers are generally restricted to the use of charged, water-soluble polymers (i.e., polyelectrolytes) ${ }^{[17]}$ and, therefore, result in films assembled through noncovalent interactions that may not be suited for applications in harsh media (e.g., extremes of $\mathrm{pH}$, ionic strength, or temperature) that could disrupt these interactions. The work reported here takes a step toward addressing several of these potential limitations by demonstrating methods for the reactive layer-bylayer assembly of suspended and covalently crosslinked multilayers directly across the open ends of pores and capillaries.

This work was motivated by recent reports demonstrating methods for the fabrication of polyelectrolyte multilayers at interfaces created between immiscible liquids. ${ }^{[27-30]}$ For example, films composed of poly(allylamine hydrochloride) and poly-(sodium 4-styrene sulfonate) have been fabricated at interfaces formed between aqueous phases and thermotropic liquid crystals. ${ }^{[28-30]}$ This approach has also been used to fabricate hollow microcapsules and nanocontainers by assembly on colloidal liquid templates. ${ }^{[27,30]}$ The results of these past studies demonstrate that solid surfaces are not a requirement for layerby-layer assembly and suggest the basis of approaches to the fabrication of suspended films and membranes (e.g., by fabricating films directly across cavities or the open ends of liquidfilled pores) using methods that would not require the physical transfer of free-standing films or chemical processes that could alter the physical or mechanical properties of the films.

We recently reported a method for the "reactive" layer-by-layer fabrication of covalently crosslinked and reactive multilayers that exploits interfacial reactions between the azlactonecontaining polymer poly(2-vinyl-4,4-dimethylazlactone) (PVDMA) and branched poly(ethylene imine) (PEI). ${ }^{[31,32]}$ The azlactone functionality of PVDMA reacts with primary amines (Scheme 1) ${ }^{[33]}$ providing a basis for both reactive/covalent assembly and the post-fabrication modification of azlactone-containing films with amine-functionalized nucleophiles. ${ }^{[31,32]}$ Because the fabrication of these materials can be performed entirely in organic solvents-including solvents that are immiscible with water-we reasoned that this approach could be used to fabricate reactive multilayers at interfaces between aqueous and organic phases.

To evaluate the feasibility of this approach, we formed aqueous/organic interfaces by hosting water within a glass capillary tube (internal diameter $\sim 1 \mathrm{~mm}$ ) and immersing one end of the capillary in dichloromethane (Fig. 1). This design was selected for our initial experiments for several reasons: i) the approach provides a convenient platform for layerby-layer assembly by simple alternate and repetitive immersion of the end of a capillary into solutions of polymer (e.g., Fig. 1), ii) it permits characterization of the morphology of films suspended across the ends of a capillary using optical, fluorescence, and scanning electron microscopy (SEM), and iii) a range of other film properties (permeability, mechanical properties, etc.) can be characterized directly using this experimental approach. 
We conducted a series of initial experiments to determine whether PEI/PVDMA films could be assembled at interfaces created between water and dichloromethane. We fabricated films directly at these interfaces by immersing the ends of water-filled capillary tubes alternately into dilute solutions of PEI or PVDMA in $\mathrm{CH}_{2} \mathrm{Cl}_{2}$ for $10 \mathrm{~s}$ (see Fig. 1). For these experiments, the opposite end of the capillary was sealed with a small drop of epoxy resin to minimize large fluctuations in the shape or level of the aqueous/organic meniscus during repetitive immersion and manipulation of the capillaries during fabrication.

Figure 2A and 2B show SEM images of the end of a capillary tube that was dipped alternately into solutions of PEI and PVDMA 30 times. For these experiments, the epoxycoated end of the capillary tube was broken off and the water within the capillary was removed to yield a suspended membrane prior to imaging. Inspection of these images reveals the presence of a uniform thin film that adheres conformally to the edges of the capillary tube and is suspended across the open end of the tube. Further inspection reveals this suspended film to be free of holes, cracks, or other large-scale defects that could form either during fabrication or as a result of the mechanical manipulation associated with the removal of water. Freely suspended PEI/ PVDMA films fabricated in this manner were stable and could be stored either dry or submerged in DMSO for at least 1 year without cracking, tearing, or detaching from the edges of the capillary.

Our past studies demonstrated that the residual azlactone functionality present in PVDMA/ PEI films fabricated on solid surfaces can be used to functionalize these films by treatment with amine-containing nucleophiles. ${ }^{[31,32]}$ We note, however, that these past studies were conducted using films fabricated entirely in organic solvents and that the two-phase conditions used in this investigation create potential problems in this context because azlactone functionality can hydrolyze in contact with water. Figure $2 \mathrm{C}$ shows a fluorescence microscopy image of the end of a capillary tube coated with a 30-bilayer film that was treated post-fabrication with the amine-functionalized fluorescent dye tetramethylrhodamine cadaverine (TMR-cad). This image reveals red fluorescence distributed uniformly across the open end of the capillary (the bright-red ring corresponds to fluorophore-functionalized film adhered to the glass surface at the end of the capillary). These results are consistent with the results of our past studies of reactive, azlactone-functionalized films fabricated on solid surfaces. ${ }^{[31,32]}$ While it is possible that some fraction of the azlactone groups of PVDMA could hydrolyze during the two-phase assembly process, it is apparent that (i) the presence of an aqueous phase during fabrication does not prevent film assembly, and (ii) that membranes assembled under these conditions contain residual azlactone functionality that can be exploited to further functionalize these materials.

Inspection of the SEM image shown in Figure 2B reveals that the film is situated taut across the end of the capillary and not deflected significantly into or out of the orifice. Additional experiments demonstrated that invaginated (i.e., concave) membranes could be fabricated by control over the relative shape of the aqueous/organic meniscus during fabrication (Fig. 2I). The application and removal of pressure to films fabricated in this manner could be used to deflect or invert suspended films into or out of the end of the capillary reversibly without fracturing the film (Fig. 2I-K; see the Supporting Information for additional details). Additional experiments will be necessary to quantify the mechanical properties of these suspended thin films. However, the results of the experiments above, when combined, demonstrate that these membranes are mechanically robust both in the dry state and when in contact with liquids. Figure $2 \mathrm{~F}$ and $2 \mathrm{G}$ shows SEM images of the end of a microcapillary tube (internal diameter $\sim 115 \mu \mathrm{m}$ ) coated with a 30-bilayer PEI/PVDMA thin film, and Figure $2 \mathrm{H}$ shows a digital image of a 30-bilayer film suspended over the large, open end of a Pasteur pipette (internal diameter $=6 \mathrm{~mm}$; the end of an untreated pipette is also shown for comparison). These results demonstrate that this general approach can be used to fabricate 
intact films suspended across the open ends of tubes ranging in size from $\sim 100 \mu \mathrm{m}$ to over $0.5 \mathrm{~cm}$ in diameter, provided that stable aqueous/organic interfaces can be maintained during fabrication.

Figure 2D shows a cross-section of an intentionally perforated 30-bilayer PEI/PVDMA film suspended across the end of a capillary tube. The top side of the film (i.e., the side of the film exposed to the dichloromethane solution during fabrication) is shown. Inspection of this image reveals a rough surface with topographical features ranging in size from tens of nanometers up to nearly $1.5 \mu \mathrm{m}$. This general morphology contrasts with that of the bottom side of the film (i.e., the side in contact with the aqueous phase during fabrication), which appears relatively smooth with much smaller surface features (Fig. 2E).We estimate the average thickness of this film to be $\sim 4.5 \mu \mathrm{m}$, as determined by measuring the point-to-point distance between the top and bottom faces of the film. We note that this value is nearly an order of magnitude larger than the thickness of 30-bilayer PEI/ PVDMA films fabricated on solid silicon substrates $(\sim 500 \mathrm{~nm})$ using entirely organic solvents, as described in our past studies. ${ }^{[32]}$ The results of additional experiments to characterize film growth profiles at these interfaces and a discussion of factors that could lead to these differences are included as Supporting Information.

Past studies have demonstrated that polyelectrolyte multilayers can be permeable to a variety of chemical and biological species..$^{[9,11,13,19,34]}$ As a result, these assemblies have been investigated as separation membranes ${ }^{[9,11]}$ and drug-carrier systems ${ }^{[13,19]}$ and are of interest in a range of fundamental contexts. ${ }^{[34]}$ We performed a series of experiments to characterize the permeability of suspended PEI/PVDMA films and to investigate the ability of these membranes to release or separate mixtures of large and/or small molecules. For these initial studies, we selected two small-molecule dyes (coumarin-152 and TMR) and the polymer PSS labeled with a rhodamine B derivative (PSS-MRho B). The molecular structures of these compounds are shown in Figure 3A. These experiments were conducted using capillaries coated with films 30 bilayers thick, identical to those described above, with the exception that the films were soaked in a solution of propylamine prior to use to react exhaustively with any residual azlactone functionality in the films and impart additional hydrophobic character. ${ }^{[31,32]}$ Capillaries were then filled with single-component solutions of each dye ( $5 \mathrm{~mm}_{\mathrm{M}}$ in DMSO), and the membrane-coated ends of the tubes were suspended in UV-vis cuvettes filled with DMSO.

Figure 3B shows a plot of solution absorbance versus time for the release of each dye from the capillary into solution. These data demonstrate that PEI/PVDMA membranes are permeable to coumarin (filled triangles), but that TMR (filled circles) and PSS-MRhoB (open squares) did not diffuse measurably across these suspended films for a period of up to 7 days. The absence of observable release in these latter cases, combined with the gradual release of coumarin over this same time period, demonstrates further the absence of tears or other large-scale defects in these films that would otherwise result in the rapid release of each of these compounds (the release of each dye from uncoated capillaries or capillaries with intentionally torn membranes occurred rapidly on the order of seconds; data not shown). The results of additional experiments using capillaries filled with 1:1 mixtures of coumarin/PSS-MRhoB or coumarin/ TMR demonstrated that these membranes can also be used as semipermeable membranes to discriminate between and separate the individual components of mixtures of large and small molecules or mixtures of two small molecules (Fig. 3C). The specific structural features (e.g., size, charge, etc.) and molecular-level interactions that lead to these large differences in permeability are not yet clear. We note, however, that the ability to functionalize the reactive azlactones in these materials with a range of functionalized amines provides opportunities to design films with permeabilities, selectivities, or other physicochemical characteristics that could be tailored to discriminate 
between or modulate the diffusion of solutes on the basis of a variety of different physical or chemical interactions (e.g., hydrophobic interactions or other specific or nonspecific supramolecular interactions, etc.).

In summary, we have reported an approach to the layer-by-layer fabrication of covalently crosslinked, reactive thin films at interfaces formed between aqueous and organic phases. This approach is based on the rapid reaction of azlactone-functionalized polymers with polymers containing primary amine functionality, and permits the direct fabrication of freely suspended and mechanically robust semipermeable membranes across the open ends of pores and orifices with dimensions on the order of tens of micrometers to several millimeters. In contrast to transfer-based approaches for assembling suspended polymer films, the approach described here should be well suited to the fabrication of suspended membranes across or within the pores, cavities, or channels of flat or topologically complex substrates (e.g., within the channels of microfluidic devices or the open ends of micropipettes). The flexibility of this approach, combined with the ability to tailor the physicochemical properties of these films post-fabrication, could thus prove useful for the fabrication of suspended films and semipermeable membranes of interest in a variety of fundamental and applied contexts.

\section{Experimental}

A detailed description of the experimental procedures can be found in the Supporting Information.

\section{Supplementary Material}

Refer to Web version on PubMed Central for supplementary material.

\section{Acknowledgments}

Financial support was provided by the NSF (DMR-0520527) through a grant to the Materials Research Science and Engineering Center (MRSEC) at the University of Wisconsin and the 3M Corporation. We thank Dr. Steven M. Heilmann (3M) and Dr. Gerald K. Rasmussen for providing samples of poly(2-vinyl-4,4-dimethylazlactone) and for many helpful discussions. We thank Nathaniel J. Fredin and Eric M. Saurer for assistance with SEM imaging. M.E.B. was funded in part by an NIH Chemistry Biology Interface Training Grant (NIGMS T32 GM008505). D.M.L. is an Alfred P. Sloan Research Fellow. Supporting Information is available online from Wiley InterScience or from the author.

\section{References}

1. Jiang CY, Markutsya S, Pikus Y, Tsukruk VV. Nat. Mater. 2004; 3:721. [PubMed: 15448680]

2. Mallwitz F, Goedel WA. Angew. Chem. Int. Ed. 2001; 40:2645.

3. Nolte M, Donch I, Fery A. ChemPhysChem. 2006; 7:1985. [PubMed: 16929555]

4. Vendamme R, Onoue SY, Nakao A, Kunitake T. Nat. Mater. 2006; 5:494. [PubMed: 16715083]

5. Gargiuli J, Shapiro E, Gulhane H, Nair G, Drikakis D, Vadgama P. J. Membr. Sci. 2006; 282:257.

6. Hisamoto H, Shimizu Y, Uchiyama K, Tokeshi M, Kikutani Y, Hibara A, Kitamori T. Anal. Chem. 2003; 75:350. [PubMed: 12553773]

7. Zhao B, Viernes NOL, Moore JS, Beebe DJ. J. Am. Chem. Soc. 2002; 124:5284. [PubMed: 11996566]

8. Bruening ML, Dotzauer DM, Jain P, Ouyang L, Baker GL. Langmuir. 2008; 24:7663. [PubMed: 18507420]

9. Merkel TC, Freeman BD, Spontak RJ, He Z, Pinnau I, Meakin P, Hill AJ. Science. 2002; 296:519. [PubMed: 11964476]

10. Nolte M, Schoeler B, Peyratout CS, Kurth DG, Fery A. Adv. Mater. 2005; 17:1665. 
11. Ulbricht M. Polymer. 2006; 47:2217.

12. Grayson ACR, Choi IS, Tyler BM, Wang PP, Brem H, Cima MJ, Langer R. Nat. Mater. 2003; 2:767. [PubMed: 14619935]

13. Mansouri S, Winnik FM, Tabrizian M. Expert Opin. Drug Delivery. 2009; 6:585.

14. Stamatialis DF, Papenburg BJ, Gironés M, Saiful S, Bettahalli SNM, Schmitmeier S, Wessling M. J. Membr. Sci. 2008; 308:1.

15. Bergbreiter DE, Liao KS. Soft Matter. 2009; 5:23.

16. Decher G. Science. 1997; 277:1232.

17. Bertrand P, Jonas A, Laschewsky A, Legras R. Macromol. Rapid Commun. 2000; 21:319.

18. Hammond PT. Adv. Mater. 2004; 16:1271.

19. Peyratout CS, Dahne L. Angew. Chem. Int. Ed. 2004; 43:3762.

20. Tang ZY, Wang Y, Podsiadlo P, Kotov NA. Adv. Mater. 2006; 18:3203.

21. Jiang CY, Kommireddy DS, Tsukruk VV. Adv. Funct. Mater. 2006; 16:27.

22. Jiang CY, Markutsya S, Tsukruk VV. Adv. Mater. 2004; 16:157.

23. Jiang CY, Tsukruk VV. Adv. Mater. 2006; 18:829.

24. Mallwitz F, Laschewsky A. Adv. Mater. 2005; 17:1296.

25. Wang Y, Angelatos AS, Caruso F. Chem. Mater. 2008; 20:848.

26. Zimnitsky D, Shevchenko VV, Tsukruk VV. Langmuir. 2008; 24:5996. [PubMed: 18457436]

27. Grigoriev DO, Bukreeva T, Mohwald H, Shchukin DG. Langmuir. 2008; 24:999. [PubMed: 18163658]

28. Gupta JK, Tjipto E, Zelikin AN, Caruso F, Abbott NL. Langmuir. 2008; 24:5534. [PubMed: 18419143]

29. Lockwood NA, Cadwell KD, Caruso F, Abbott NL. Adv. Mater. 2006; 18:850.

30. Tjipto E, Cadwell KD, Quinn JF, Johnston APR, Abbott NL, Caruso F. Nano Lett. 2006; 6:2243. [PubMed: 17034091]

31. Buck ME, Breitbach AS, Belgrade SK, Blackwell HE, Lynn DM. Biomacromolecules. 2009; 10:1564. [PubMed: 19438231]

32. Buck ME, Zhang J, Lynn DM. Adv. Mater. 2007; 19:3951.

33. Heilmann SM, Rasmussen JK, Krepski LR. J. Polym. Sci. Part A. 2001; 39:3655.

34. Johnston APR, Caruso F. J. Am. Chem. Soc. 2005; 127:10014. [PubMed: 16011361] 

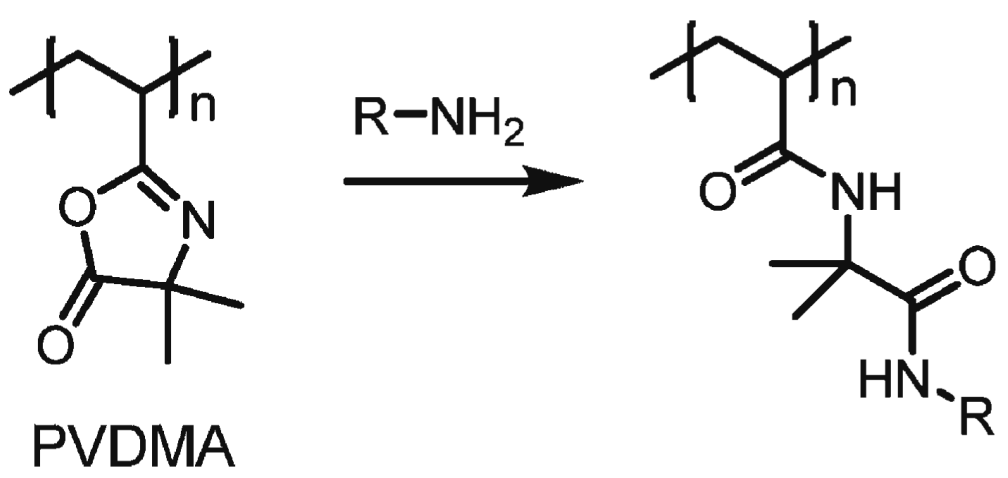

Scheme 1.

Schematic representation of the reaction of a primary amine-functionalized compound with an azloactone-functionalized polymer 

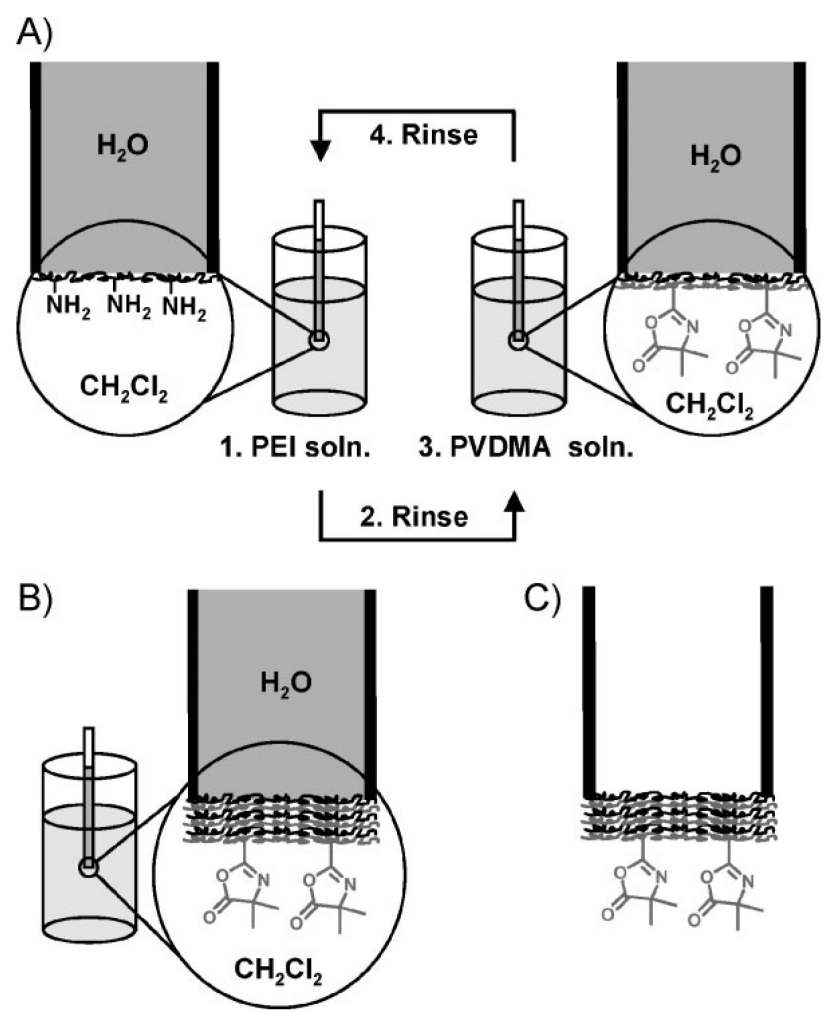

Figure 1.

Illustration depicting reactive layer-by-layer fabrication of suspended thin films at aqueous/ organic interfaces. A) The end of a water-filled glass capillary is dipped briefly into a solution of PEI dissolved in dichloromethane. Subsequent immersion into a solution of PVDMA facilitates reactive deposition of a thin layer of PVDMA. B) Repetition of this process results in the assembly of covalently crosslinked multilayers across the end of the capillary. C) Removal of the water from within the capillary tube results in a reactive thin film suspended across the end of the capillary. (For a color version of this figure, see Figure $\mathrm{S} 2$ of the Supporting Information.) 

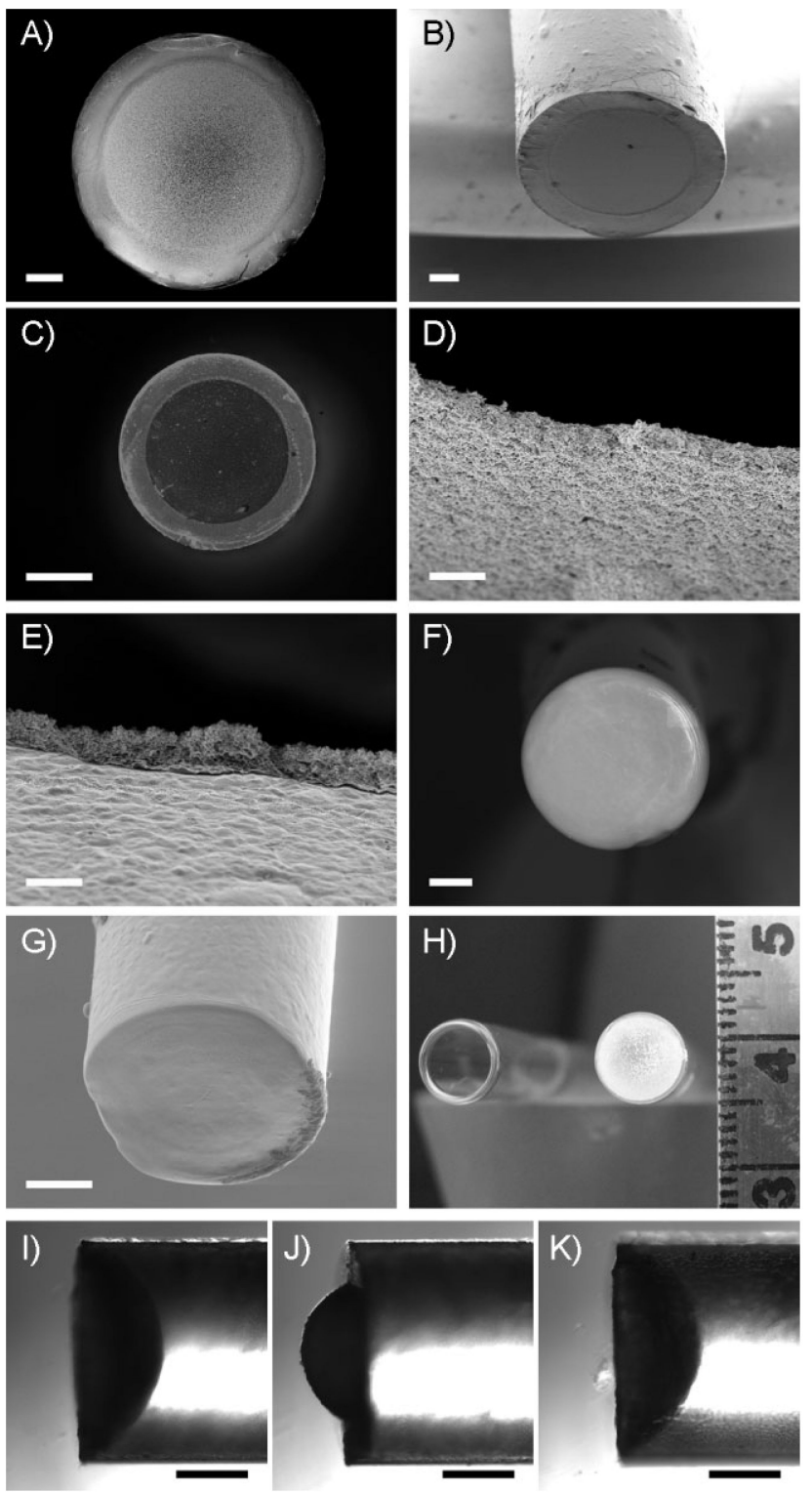

Figure 2.

A,B) SEM images showing the end of a glass capillary tube coated with an thin PEI/ PVDMA film. C) Fluorescence microscopy image of the end of a coated capillary tube after treatment with TMR-cad. D,E) SEM images of a cross-section of a film fabricated over the end of a capillary tube that was intentionally perforated prior to imaging, showing (D) the side of the film exposed to organic solutions during fabrication and $(\mathrm{E})$ the side of the film exposed to the aqueous phase during fabrication. F,G) SEM images of the end of a microcapillary coated with a 30-bilayer PEI/PVDMA film. H) Digital photograph of a film fabricated over the large end of a Pasteur pipette (right); an uncoated pipette (left) is included for comparison. I) Side-on, phase-contrast microscopy image of the end of a capillary tube coated with an invaginated film fabricated by control over the shape of the aqueous/organic meniscus during fabrication (see text). J) Image of the film depicted in (I) shown to be protruding, or inverted, after the application of pressure to the open end of a water-filled capillary. K) Removal of pressure from the film shown in (J) results in deflection of the film back into the capillary. Scale bars correspond to $200 \mu \mathrm{m}(\mathrm{A}, \mathrm{B}), 500$ 
$\mu \mathrm{m}(\mathrm{C}), 10 \mu \mathrm{m}$ (D,E), $40 \mu \mathrm{m}(\mathrm{F}), 50 \mu \mathrm{m}(\mathrm{G})$, scale in $\mathrm{cm}(\mathrm{H}), 500 \mu \mathrm{m}(\mathrm{I}-\mathrm{K})$. (For a color version of this figure, see Figure $\mathrm{S} 3$ of the Supporting Information.) 
A)
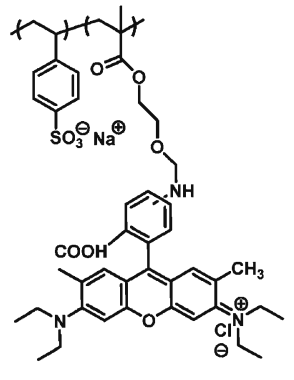

SPS-MRho B

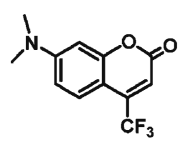

Coumarin 152

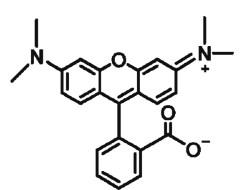

Tetramethylrhodamine

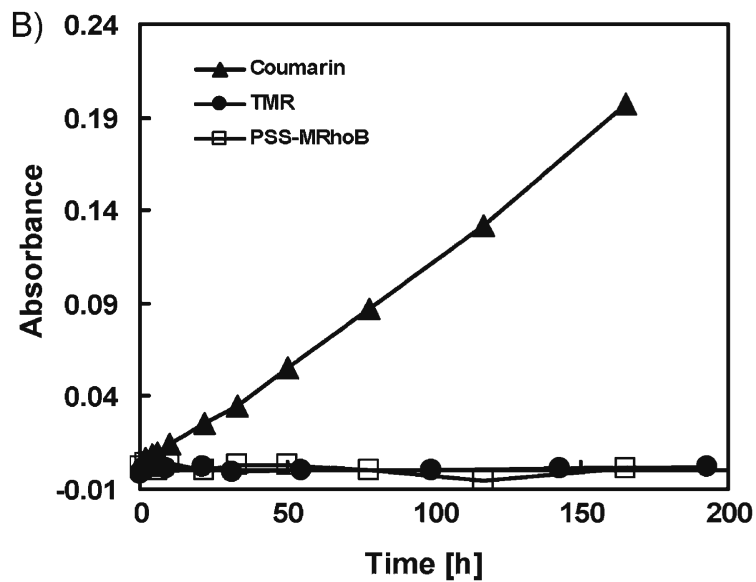

C)

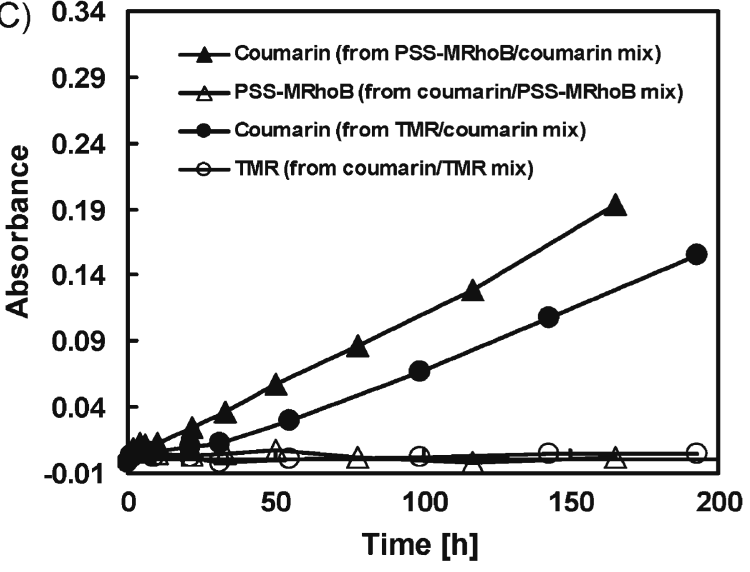

Figure 3.

A) Chemical structures of the fluorescent molecules used in diffusion/release experiments. B) Plot of solution absorbance (measured using UV-vis) over time for capillaries filled with solutions of coumarin (filled triangles), TMR (filled circles), or PSS-MRhoB (open squares). Experiments were performed at $37^{\circ} \mathrm{C}$ using capillaries with open ends coated with a PEI/ PVDMA membrane 30 bilayers thick. C) Plot of solution absorbance versus time for the release from capillaries filled with two-component mixtures of coumarin, TMR, or PSSMRhoB (see legend). 